Acta Crystallographica Section B

Structural

Science

ISSN 0108-7681

Samantha Y. Chong, ${ }^{\mathrm{a}}$ Colin C. Seaton, ${ }^{\mathrm{b}}$ Benson M. Kariuki ${ }^{\mathrm{a}}$ and Maryjane Tremayne ${ }^{\mathrm{a} *}$

aschool of Chemistry, University of Birmingham, Edgbaston, Birmingham B15 2TT, England, and b Institute of Pharmaceutical Innovation, School of Pharmacy, University of Bradford, Bradford BD7 1DP, England

Correspondence e-mail:

m.tremayne@bham.ac.uk

\title{
Molecular versus crystal symmetry in tri-substituted triazine, benzene and isocyanurate derivatives
}

The crystal structures of triethyl-1,3,5-triazine-2,4,6-tricarboxylate (I), triethyl-1,3,5-benzenetricarboxylate (II) and tris2-hydroxyethyl isocyanurate (III) have been determined from conventional laboratory X-ray powder diffraction data using the differential evolution structure solution technique. The determination of these structures presented an unexpectedly wide variation in levels of difficulty, with only the determination of (III) being without complication. In the case of (I) structure solution resulted in a Rietveld refinement profile that was not ideal, but was subsequently rationalized by single-crystal diffraction as resulting from disorder. Refinement of structure (II) showed significant variation in sidechain conformation from the initial powder structure solution. Further investigation showed that the structure solution optimization had indeed been successful, and that preferred orientation had a dramatic effect on the structure-solution $R$ factor search surface. Despite the presence of identical side chains in (I) and (II), only the triazine-based system retains threefold molecular symmetry in the crystal structure. The lack of use of the heterocyclic $\mathrm{N}$ atom as a hydrogen-bond acceptor in this structure results in the formation of a similar non-centrosymmetric network to the benzene-based structure, but with overall three-dimensional centrosymmetry. The hydrogen-bonded layer structure of (III) is similar to that of other isocyanurate-based structures of this type.

\section{Introduction}

The control of solid-state supramolecular synthesis and the design of functionalized materials is the ultimate aim of any crystal engineering strategy. However, the incorporation of desirable structural features through the transfer of molecular symmetry to crystal symmetry is an element of crystal structure design that is often overlooked. One area in which this aspect has been successful is in the design of octupolar nonlinear optical (NLO) materials based on a series of isocyanurate and triazine compounds with $C_{3}$ molecular symmetry (Thalladi et al., 1997, 1998, 1999). A feature of these materials is the formation of a two-dimensional trigonal noncentrosymmetric network that is characterized by orientation of the molecules within the plane such that the alternating 'unlike' ring substituents are pointing directly towards each other (Thalladi et al., 1998). In the majority of these structures $C_{3}$ molecular symmetry is retained in the crystal packing, although structures with reduced symmetry can also form this distinctive trigonal network (Thalladi et al., 1997). The construction of this 'local' acentric structural feature is an important intermediate stage in the design of these materials (Panunto et al., 1987), but the non-centrosymmetry must be
Received 17 February 2006
(C) 2006 International Union of Crystallography Printed in Great Britain - all rights reserved \author{
Accepted 1 June 2006
}


extended into the bulk structure in order for the material to display NLO properties such as second harmonic generation.

As part of our on-going research programme into the development of structure solution methods from powder diffraction data, we have studied a series of similar materials all based on a central isocyanurate, triazine or benzene unit and potentially displaying $C_{3}$ molecular symmetry. Here we report the structure determination of three such compounds, triethyl-1,3,5-triazine-2,4,6-tricarboxylate (I), triethyl-1,3,5benzenetricarboxylate (II) and tris-2-hydroxyethyl isocyanurate [also known as 1,3,5-tris(2-hydroxyethyl)cyanuric acid] (III) (see scheme) from laboratory X-ray powder diffraction

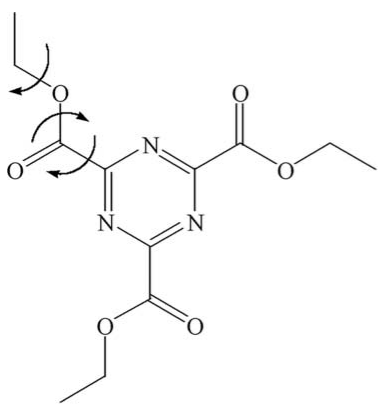

(I)

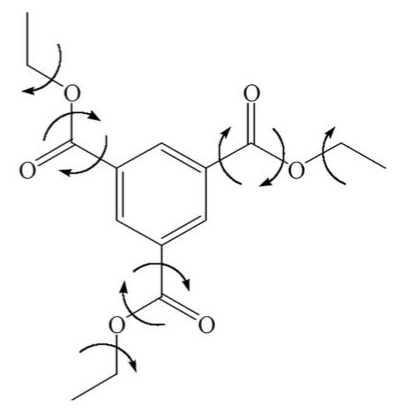

(II)

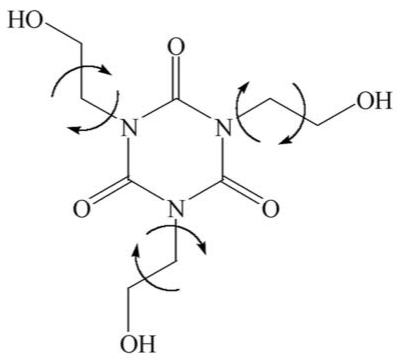

(III)

data. All three materials contain flexible side chains and can therefore either retain their molecular symmetry in the crystal structure or reduce their symmetry through conformational variation. The presence of identical ethyl carboxylate side chains in (I) and (II) also enables us to make a direct comparison of the triazine- and benzene-based structures, with the absence of any strong hydrogen-bond donors giving an insight into the influence of weak hydrogen bonding on the crystal packing in these compounds. Although few previous comparisons have been made between other analogous systems, distinct differences in crystal structure are observed, with the central triazine or benzene unit playing a defining role in supramolecular packing; i.e. the dominant $\mathrm{CH} \cdots \mathrm{N}$ planar layer network in 2,4,6-triethynyl-1,3,5-triazine (Ohkita et al., 2002) is replaced by a $\mathrm{CH} \cdots \pi$ folded layer structure in 1,3,5-triethynylbenzene (Weiss et al., 1997), whereas the difference in packing density between the tris-dithiadiazolyl derivatives 1,3,5- $\mathrm{C}_{6} \mathrm{H}_{3}\left(\mathrm{CN}_{2} \mathrm{~S}_{2}\right)_{3}$ and $\mathrm{C}_{3} \mathrm{~N}_{3}\left(\mathrm{CN}_{2} \mathrm{~S}_{2}\right)_{3}$, and the use of a more symmetrical packing motif in the triazine-based structure, can be attributed directly to the contrast between
$\mathrm{N} \cdots \mathrm{S}$ and $\mathrm{CH} \ldots \mathrm{S}$ buffering interactions between neighbouring molecules (Cordes et al., 1993).

The structure determination of molecular materials from powder diffraction data is now becoming an established but, as illustrated in this paper, not a routine process. The majority of molecular structures are solved using direct-space structure solution methods (Harris et al., 2001; David et al., 2002; Tremayne, 2004), which generate trial crystal structures utilizing molecular connectivity in the structure solution calculation, and assess the fitness of each structure by direct comparison between its calculated diffraction pattern and the experimental data. Global optimization methods are used to guide the calculation locating the minimum of the search hypersurface corresponding to the structure solution. A number of different optimization methods have been used in structure solution from powder data, including both sequential and evolutionary algorithms (Newsam et al., 1992; Harris et al., 1994; Andreev et al., 1996; Kariuki et al., 1997; David et al., 1998; Cheung et al., 2002; Favre-Nicolin \& Cerny, 2002; Johnston et al., 2002; Pagola et al., 2000). In this paper we have used an evolutionary algorithm based on differential evolution (DE) optimization (Price, 1999), a technique that has been applied successfully to the structure determination of several molecular materials from powder diffraction data (Seaton \& Tremayne, 2002a; Tremayne et al., 2002b). DE is a relatively new algorithm that creates new members of the population by recombination and mutation of randomly selected members of the current population, forming a new generation via a purely deterministic method of selection. The recombination and mutation processes are carried out in a single step, with the levels of each term controlled using parameters $K$ and $F$, respectively (Tremayne et al., 2002b). Adjustment of the $K$ and $F$ parameters (taking values between 0 and 1 ) enables straightforward control of the search dynamic, the only other user-defined parameter being population size. The small number of optimization parameters means that this is a simple method to use and implement, while offering a robust searching of minima with the algorithm adapting to the hypersurface as time proceeds.

As described elsewhere (Harris et al., 2001; Tremayne, 2004), the complexity of a direct-space structure solution calculation depends predominantly on the number of variables required to define the structure (i.e. the number of independent molecules or the degree of conformational flexibility) rather than the number of atoms in the asymmetric unit. Hence, in principle, the complexity of structure solution for the three compounds (I)-(III) should be comparable, with the only significant differences arising from symmetry considerations. However, as detailed below, the determination of these three structures from powder diffraction data presented us with widely varying levels of difficulty, with only the structure determination of (III) being without complication. Despite the optimization process in the structure solution of (I) being made significantly easier through the retention of symmetry within the molecule, the effects of disorder led us to the use of single-crystal diffraction for full rationalization of the structure and confirmation that the initial powder struc- 
Table 1

Experimental details - powder data.

\begin{tabular}{|c|c|c|c|}
\hline & (I) & (II) & (III) \\
\hline \multicolumn{4}{|l|}{ Crystal data } \\
\hline Chemical formula & $\mathrm{C}_{12} \mathrm{H}_{15} \mathrm{~N}_{3} \mathrm{O}_{6}$ & $\mathrm{C}_{15} \mathrm{H}_{18} \mathrm{O}_{6}$ & $\mathrm{C}_{9} \mathrm{H}_{15} \mathrm{~N}_{3} \mathrm{O}_{6}$ \\
\hline$M_{r}$ & 297.27 & 294.31 & 261.24 \\
\hline Cell setting, space group & Hexagonal, $\mathrm{P6}_{3} / m$ & Hexagonal, $P 6_{1}$ & Monoclinic, $P 2_{1} / n$ \\
\hline Temperature $(\mathrm{K})$ & $293(2)$ & $293(2)$ & $293(2)$ \\
\hline$a, b, c(\AA)$ & $10.9830(3), 10.9830(3), 6.7555(2)$ & $\begin{array}{c}11.3588(2)[11.3438(17)], \dagger \\
11.3588(2)[11.3438(17)], \dagger \\
20.2725(4)[19.665(4)] \dagger\end{array}$ & $10.4105(3), 13.1294(5), 8.6735$ (3) \\
\hline$\beta$ & 90 & 90 & $98.222(2)$ \\
\hline Final $V\left(\AA^{3}\right)$ & $705.72(5)$ & $2265.18(7)[2191.5(6)] \dagger$ & $1173.34(9)$ \\
\hline$Z$ & 2 & 6 & 4 \\
\hline$D_{x}\left(\mathrm{Mg} \mathrm{m}^{-3}\right)$ & 1.399 & 1.295 & 1.479 \\
\hline Radiation type, wavelength $(\AA)$ & $\mathrm{Cu} K \alpha_{1}, 1.54056$ & $\mathrm{Cu} K \alpha_{1}, 1.54056$ & $\mathrm{Cu} K \alpha_{1}, 1.54056$ \\
\hline$\mu\left(\mathrm{mm}^{-1}\right)$ & 0.973 & 0.843 & 1.078 \\
\hline Specimen form, colour & Powder, white & Powder, white & Powder, white \\
\hline \multicolumn{4}{|l|}{ Data collection } \\
\hline Diffractometer & $\begin{array}{l}\text { Bruker AXS D5000 with PSD } \\
\quad\left(\text { covering } 8^{\circ} \text { in } 2 \theta\right)\end{array}$ & $\begin{array}{l}\text { Bruker AXS D5000 with PSD } \\
\quad\left(\text { covering } 8^{\circ} \text { in } 2 \theta\right)\end{array}$ & $\begin{array}{l}\text { Bruker AXS D5000 with PSD } \\
\quad\left(\text { covering } 8^{\circ} \text { in } 2 \theta\right)\end{array}$ \\
\hline Data collection method & $\begin{array}{l}\text { Disc geometry; transmission mode; } \\
\text { step scan }\end{array}$ & $\begin{array}{l}\text { Disc geometry; transmission mode; } \\
\text { step scan }\end{array}$ & $\begin{array}{l}\text { Disc geometry; transmission mode; } \\
\text { step scan }\end{array}$ \\
\hline $\begin{array}{l}2 \theta \text { range min-max, increment }\left({ }^{\circ}\right), \\
\text { total time }(h)\end{array}$ & $10-50,0.02,1$ & $4-80,0.02,15$ & $10-70,0.02,1$ \\
\hline \multicolumn{4}{|l|}{ Structure solution } \\
\hline $\begin{array}{l}\text { Le Bail } R \text { factors, } R_{\mathrm{wp}} \\
\quad \text { goodness of fit, } S\end{array}$ & $0.049 ; \ddagger 1.40$ & $0.049 ; 4.52$ & $0.059 ; 1.34$ \\
\hline DE Elements & 4 & 15 & 12 \\
\hline Population size & 60 & 300 & 120 \\
\hline$K$ & 0.99 & 0.99 & 0.99 \\
\hline Best $F$ & 0.4 & 0.3 & 0.5 \\
\hline Average $R_{\mathrm{wp}}$ & 0.425 & 0.300 & 0.347 \\
\hline Best $R_{\mathrm{wp}}$ & 0.141 & 0.130 & 0.099 \\
\hline \multicolumn{4}{|l|}{ Refinement } \\
\hline Refinement on & Full-matrix least-squares on $F^{2}$ & Full-matrix least-squares on $F^{2}$ & Full-matrix least-squares on $F^{2}$ \\
\hline$R$ factors, $R_{p}, R_{\mathrm{wp}}$; goodness of fit, $S$ & $0.042,0.067 ; 1.90$ & $0.042,0.058 ; 5.36$ & $0.049,0.065 ; 1.48$ \\
\hline No. of reflections & 496 & 493 & 515 \\
\hline No. of parameters & 36 & 143 & 105 \\
\hline No. of restraints & 32 & 110 & 89 \\
\hline $\begin{array}{l}\text { Preferred orientation fraction } \\
\text { [and direction] }\end{array}$ & $0.658[110]$ & $0.749[010]$ & 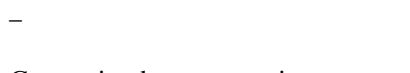 \\
\hline H-atom treatment & Constrained to parent site & Constrained to parent site & Constrained to parent site \\
\hline$(\Delta / \sigma)_{\max }$ & $<0.0001$ & $<0.0001$ & $<0.0001$ \\
\hline
\end{tabular}

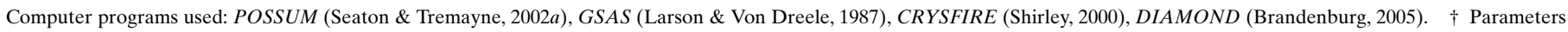
from low-temperature single-crystal study (Dale \& Elsegood, 2003). $\quad$ 末 DE structure solution in $P 6_{3}$.

ture was indeed correct. The structure determination of (II) from powder diffraction data has been previously reported (Tremayne et al., 2002a), although neither a description of the crystal structure or details of the structure solution calculation were given. In this case significant changes in side-chain conformation were observed during refinement, the implications of which will be discussed later. We present our results from a more detailed investigation of this structure solution calculation and the $R$ factor hypersurface involved, showing the effect that factors such as preferred orientation or choice of structural model can have on success. The crystal structure of (II) has since been redetermined, and confirmed within experimental error, at $150 \mathrm{~K}$, using single-crystal diffraction methods (Dale \& Elsegood, 2003).

\section{Structure solution and refinement}

Samples of (I)-(III) were purchased from Aldrich (UK) and used directly as received. Experimental details of the powder [for (I)-(III)] and single-crystal X-ray diffraction data collection [for (I)] are given in Tables 1 and 2, respectively. ${ }^{1}$ For both (I) and (II), a number of powder diffraction data sets were collected in both transmission disc and capillary geometry, with the variation in relative intensities between these data sets identifying the preferred orientation as a major consideration. The data sets used for structure characteriza-

\footnotetext{
${ }^{1}$ Supplementary data for this paper are available from the IUCr electronic archives (Reference: BM5031). Services for accessing these data are described at the back of the journal.
} 
are shown in Fig. 1 and the final agreement factors from refinement are given in Table 1 .

2.1.2. Single-crystal X-ray diffraction. The data were processed using SAINT-Plus (Bruker, 2001), and the structure of (I) was solved and refined using SHELXL97 (Sheldrick, 1997). The non-H atoms were refined anisotropically, whereas the $\mathrm{H}$ atoms were placed in calculated positions and refined using a riding model, with atomic displacement parameters of

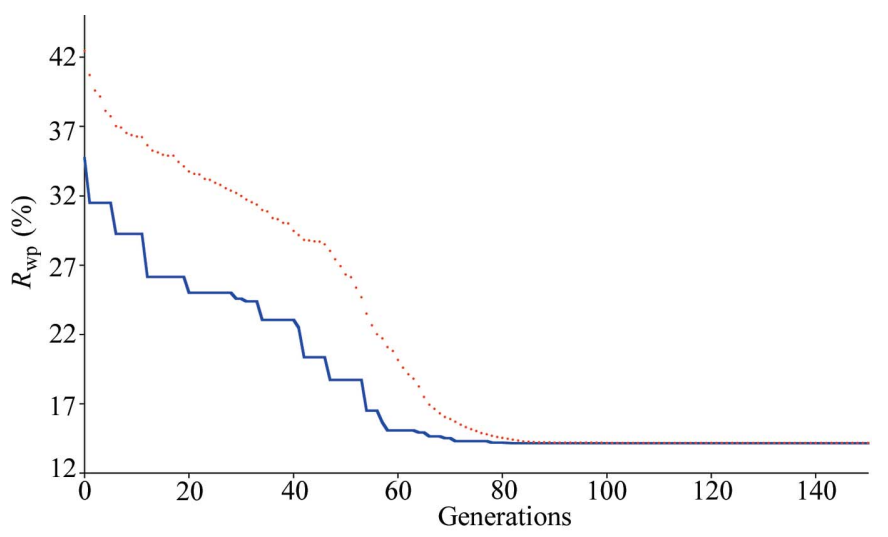

(a)

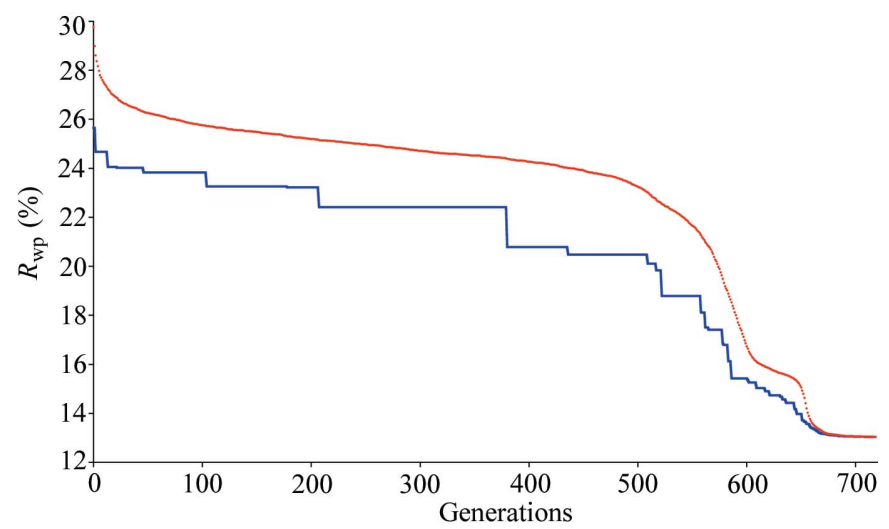

(b)

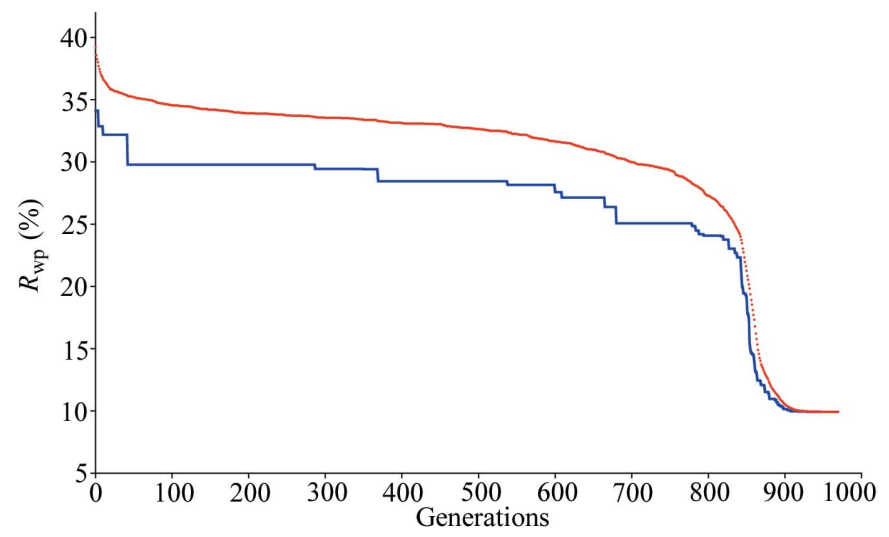

(c)

Figure 2

Differential evolution progress plot for the structure solution of $(a)$ (I), (b) (II) and (c) (III) showing the best $R_{\mathrm{wp}}$ (line) and mean $R_{\mathrm{wp}}$ (open circles) for a DE calculation.
1.2 times those of the atoms they are bonded to. Further details are given in Table 2.

\subsection{Details of structure solution}

2.2.1. Triethyl-1,3,5-triazine-2,4,6-tricarboxylate (I). In the case of (I) both $P 6_{3}$ and $P 6_{3} / m$ were identified as possible space groups, although both required the imposition of symmetry constraints, i.e. location of the molecule around the $\left(\frac{1}{3}, \frac{2}{3}, z\right)$ axis in $P 6_{3}$ or with additional mirror symmetry in $\mathrm{P6}_{3} / m$ (on a -6 site). High-resolution solid-state ${ }^{13} \mathrm{C} \mathrm{NMR}$ spectroscopy confirmed the presence of threefold symmetry within the molecule, indicating only four crystallographically distinct carbon environments. Structure solution was initially attempted in the lower-symmetry $P 6_{3}$, so as to minimize the use of constraints and avoid possible imposition of incorrect symmetry. The structural model of (I) used in the DE calculation comprised a third of the molecule constructed using standard bond lengths and angles, excluding the methyl $\mathrm{H}$ atoms. Structure solution required rotation of the structural model around the $\left(\frac{1}{3}, \frac{2}{3}, z\right)$ axis, with conformational flexibility described by three freely rotating bonds, as shown in the scheme. Thus, each member of the population consisted of four DE elements, with the population size fixed at 60 members. Five DE calculations were carried out, with $K=0.99$ and $F=0.4$. All five runs converged to within $0.2 \%$ of the $R_{\mathrm{wp}}$ of the best solution, which was clearly distinguishable from the average random structures generated in the DE calculation (Table 1 and Fig. 2a). The conformation of this solution had both the carboxyl and alkyl parts of the flexible chain in the plane of the central aromatic ring (with the alkyl $\mathrm{H}$ atoms staggered above and below), suggesting that the correct space group is $P 6_{3} / m$ with the molecule lying on a mirror plane. The structure was translated onto the mirror plane at the -6 site and refined in $P 6_{3} / m$ as described in $\$ 2.1 .1$.

It is clear from Fig. 1(a) that the fit of this crystal structure to the X-ray powder diffraction data is not ideal. Fortunately, the sample contained some small crystals of suitable quality

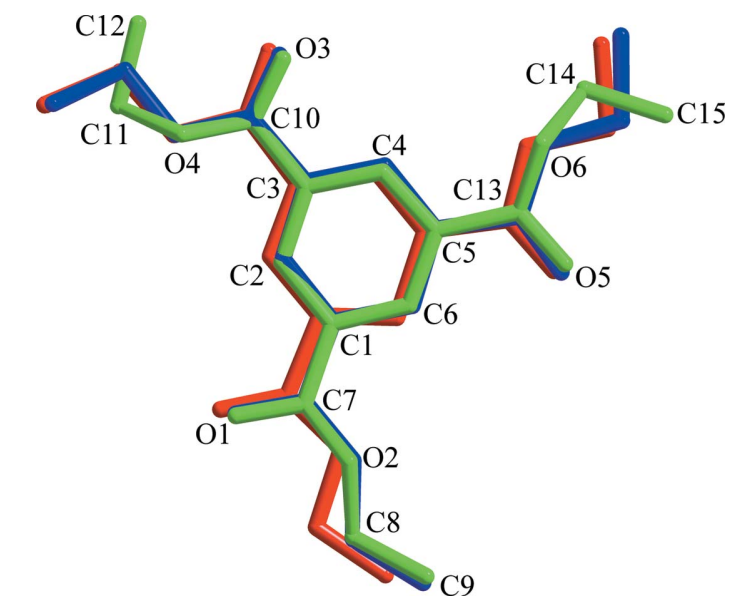

Figure 3

The molecular conformation of (II) from the DE solution (green), the final refined structure (blue) and the published single-crystal structure (red). The atom-labelling scheme is also shown. 
for structure determination from single-crystal X-ray diffraction data. This subsequent analysis identified the presence of potential disorder in the structure (\$3.1.1), and clearly confirmed that the crystal packing obtained by powder methods was correct (the minimum, maximum and mean distances between pairs of corresponding atoms in the singlecrystal and powder structures are $0.05,0.16$ and $0.10 \AA$ respectively). However, in combination with the effects of preferred orientation and inhomogeneity in this sample, we were unable to model the disorder sufficiently well to improve the Rietveld profile.

2.2.2. Triethyl-1,3,5-benzenetricarboxylate (II). The structural model of (II) used for structure solution comprised the complete molecule constructed using standard bond lengths and angles, excluding the methyl $\mathrm{H}$ atoms. Hence, structure solution required variation of 15 structural parameters: three for translation and three for orientation of the molecule in the unit cell, with the remaining nine parameters used to describe the conformation of the three side chains as shown in the scheme. Thus, each member of the population consisted of 15 DE elements with the population size fixed at 300 members. Five DE calculations were carried out, with $K=0.99$ and $F=$ 0.3 , four of which resulted in the same solution, with a significantly lower $R_{\mathrm{wp}}$ value than other structures generated during the DE calculation (Table 1 and Fig. $2 b$ ). This structure solution was used as a starting point for successful Rietveld refinement (Fig. 1b). The resulting structure is in good agreement with that obtained from the subsequent lowtemperature single-crystal diffraction study (Dale \& Elsegood, 2003; Fig. 3), given the significant temperature difference between the two studies (the minimum, maximum and mean distances between pairs of corresponding atoms are $0.27,0.82$ and $0.50 \AA$, respectively).

However, comparison of the molecular geometry of the structure obtained from the DE calculation and that in the final refined structure shows significant deviation in conformation in two of the side chains (Fig. 3). This deviation can be quantified by consideration of the difference $(\Delta)$ in torsion angle values between the two structural models; $\Delta(\mathrm{C} 5-$ $\mathrm{C} 13-\mathrm{O} 6-\mathrm{C} 14)=70^{\circ}, \Delta(\mathrm{C} 13-\mathrm{O} 6-\mathrm{C} 14-\mathrm{C} 15)=93^{\circ}$, $\Delta(\mathrm{C} 3-\mathrm{C} 10-\mathrm{O} 4-\mathrm{C} 11)=24^{\circ}$ and $\Delta(\mathrm{C} 10-\mathrm{O} 4-\mathrm{C} 11-\mathrm{C} 12)=$ $110^{\circ}$. To investigate whether these differences in molecular conformation arise from differences in the solution and refinement hypersurfaces and to confirm that the DE calculation had indeed located its global minimum, a series of grid search calculations were carried out to enable complete

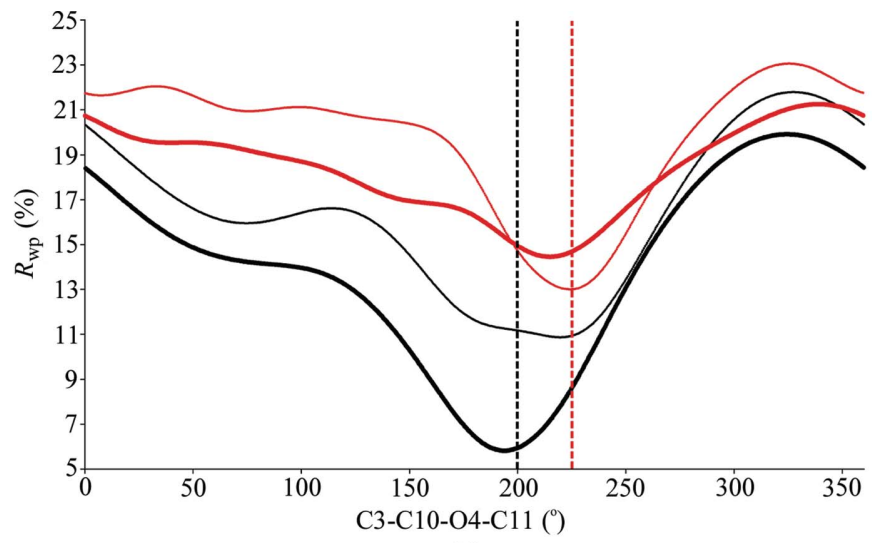

(a)

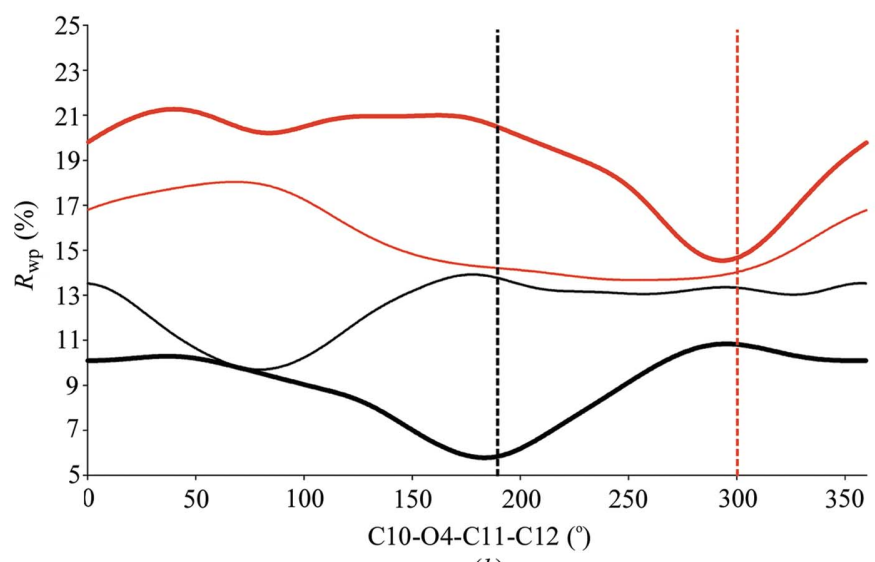

(b)

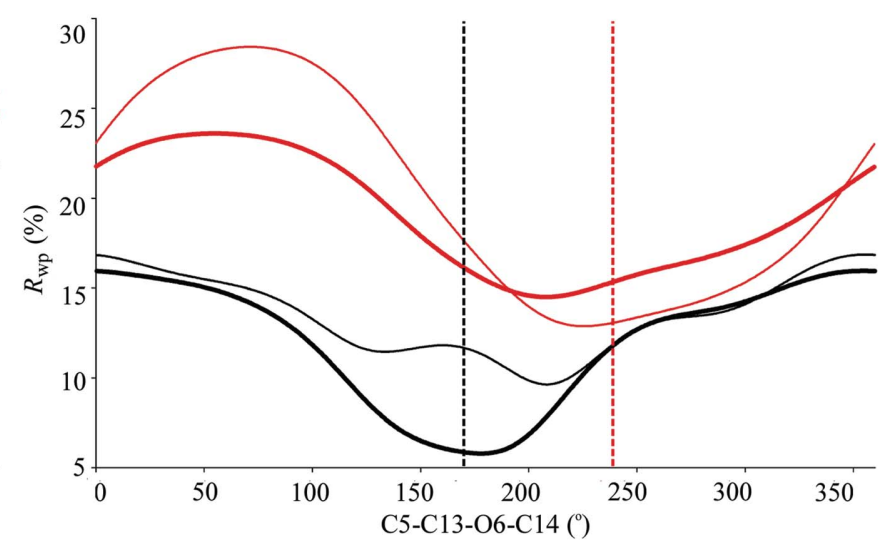

(c)

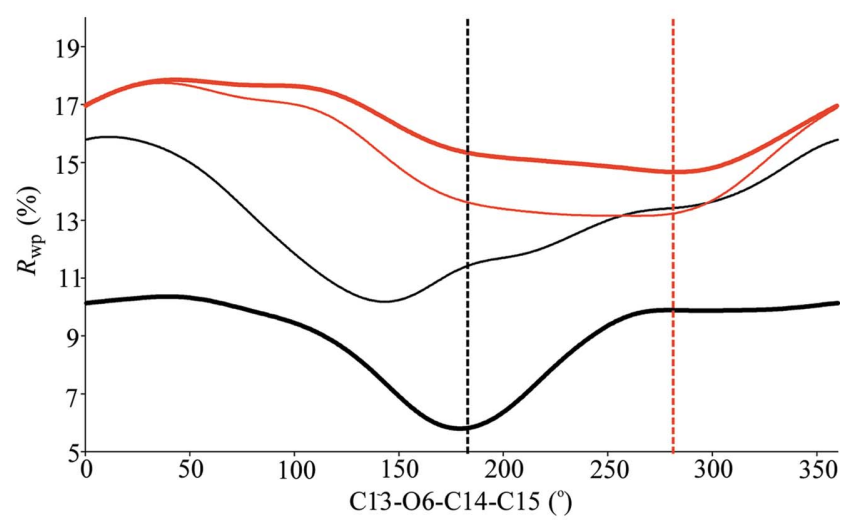

(d)

Figure 4

$R_{\mathrm{wp}}$ versus torsion angle for $(a) \mathrm{C} 3-\mathrm{C} 10-\mathrm{O} 4-\mathrm{C} 11,(b) \mathrm{C} 10-\mathrm{O} 4-\mathrm{C} 11-\mathrm{C} 12,(c) \mathrm{C} 5-\mathrm{C} 13-\mathrm{O} 6-\mathrm{C} 14$ and $(d) \mathrm{C} 13-\mathrm{O} 6-\mathrm{C} 14-\mathrm{C} 15$ in structure (II). The red curves show the DE hypersurface and black curves the Rietveld hypersurface, both with (thick lines) and without (thin lines) the preferred orientation correction applied (preferred orientation parameter $=0.749$ or 1.000 , respectively). Vertical dashed lines indicate the torsion angle located in the DE (red) and final refined (black) structural models. 
Table 3

Selected intramolecular torsion angles $\left(^{\circ}\right.$ ) for (I)-(III).

\begin{tabular}{lrlrlr}
\hline Compound (I) & & & & \\
N1-C1-C2-O3 & 0 & $\mathrm{C} 1-\mathrm{C} 2-\mathrm{O} 3-\mathrm{C} 4$ & 180 & $\mathrm{C} 2-\mathrm{O} 3-\mathrm{C} 4-\mathrm{C} 5$ & 180 \\
& & & & & \\
Compound (II) & & & & \\
$\mathrm{C} 6-\mathrm{C} 1-\mathrm{C} 7-\mathrm{O} 2$ & $-2.7(3)$ & $\mathrm{C} 1-\mathrm{C} 7-\mathrm{O} 2-\mathrm{C} 8$ & $-178.6(2)$ & $\mathrm{C} 7-\mathrm{O} 2-\mathrm{C} 8-\mathrm{C} 9$ & $174.6(2)$ \\
$\mathrm{C} 2-\mathrm{C} 3-\mathrm{C} 10-\mathrm{O} 4$ & $3.0(3)$ & $\mathrm{C} 3-\mathrm{C} 10-\mathrm{O} 4-\mathrm{C} 11$ & $178.4(2)$ & $\mathrm{C} 10-\mathrm{O} 4-\mathrm{C} 11-\mathrm{C} 12$ & $-172.3(2)$ \\
$\mathrm{C} 4-\mathrm{C} 5-\mathrm{C} 13-\mathrm{O} 6$ & $1.2(3)$ & $\mathrm{C} 5-\mathrm{C} 13-\mathrm{O}-\mathrm{C} 14$ & $-178.7(2)$ & $\mathrm{C} 13-\mathrm{O} 6-\mathrm{C} 14-\mathrm{C} 15$ & $172.0(2)$ \\
& & & & & \\
Compound (III) & & & & & \\
C2-N1-C1-C11 & $-89(1)$ & $\mathrm{C} 6-\mathrm{N} 5-\mathrm{C} 5-\mathrm{C} 51$ & $-104(1)$ & $\mathrm{N} 3-\mathrm{C} 3-\mathrm{C} 31-\mathrm{O} 32$ & $61(1)$ \\
$\mathrm{C} 4-\mathrm{N} 3-\mathrm{C} 3-\mathrm{C} 31$ & $83(1)$ & $\mathrm{N} 1-\mathrm{C} 1-\mathrm{C} 11-\mathrm{O} 12$ & $-179(1)$ & $\mathrm{N} 5-\mathrm{C} 5-\mathrm{C} 51-\mathrm{O} 52$ & $69(1)$ \\
\hline
\end{tabular}

the DE calculations, $(e)$ there is a distinct difference between the position of the minima in the DE and refinement surfaces when compared both before and after the preferred orientation correction has been applied. These observations clearly illustrate the drastic effect that both slight movement and relaxation of molecular geometry, and other factors such as preferred orientation (and the associated shift of global minima), can have on successful structure determination, highlighting the need for a full Rietveld

construction and visualization of these hypersurfaces. As optimization techniques such as DE are efficient search algorithms, a separate grid search was needed for systematic variation of the side chains under consideration, i.e. intramolecular rotation about the $\mathrm{C} 13-\mathrm{O} 6, \mathrm{O} 6-\mathrm{C} 14, \mathrm{C} 10-\mathrm{O} 4$ and $\mathrm{O} 4-\mathrm{C} 11$ bonds, respectively, so that both the relevant solution and refinement hypersurfaces could be fully explored. Using the DE structure solution and the final refined structure as starting models, each torsion angle was rotated independently in steps of $0.5^{\circ}$, with the rest of the molecule unchanged. These calculations were performed with and without the inclusion of the final refined preferred orientation correction to assess the effect of this parameter on the hypersurface (Fig. 4).

These figures illustrate a number of important factors that should be taken into consideration during the structure determination process and explain the behaviour of this structure during refinement: $(a)$ the DE calculation is successfully locating the minima of the hypersurface that it searches, although some of the minima associated with the rotation of the end groups are broad and ill-defined, $(b)$ introduction of a preferred orientation parameter in the DE calculation raises the $R_{\mathrm{wp}}$ values of the minima but does not have a significant effect on their positions, $(c)$ inclusion of preferred orientation in refinement results in an overall hypersurface with lower $R_{\mathrm{wp}}$ (as expected), but with a significant shift in the position of the minima, $(d)$ the minima in each refinement hypersurface are sharper than those seen in

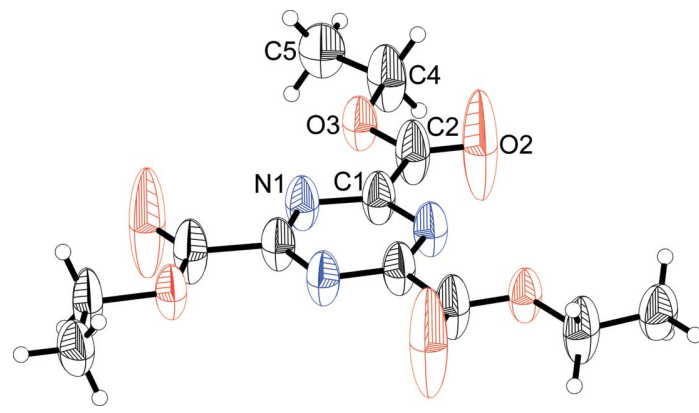

Figure 5

An ORTEPIII (Burnett \& Johnson, 1996) view of (I), showing the atomlabelling scheme. Displacement ellipsoids are drawn at the $50 \%$ probability level and $\mathrm{H}$ atoms are shown as small spheres of arbitrary radii. refinement after structure solution.

2.2.3. Tris-2-hydroxyethyl isocyanurate (III). The structural model of (III) used for structure solution was constructed in a similar way to that for (II), excluding the hydroxyl $\mathrm{H}$ atoms, such that six freely rotating bonds were needed to describe the conformational flexibility of the molecule (see scheme). The lack of crystallographic symmetry in this molecule was confirmed using high-resolution solid-state ${ }^{13} \mathrm{C}$ NMR spectroscopy, which clearly showed distinct peaks for each C-atom environment. Structure solution required consideration of 12 elements and a population size of 120 was used. The DE calculation was run five times using the control parameters $K=$ 0.99 and $F=0.5$, and returned a clearly distinguishable solution (Table 1 and Fig. 2c). This structure was then successfully refined as described earlier (Fig. 1c).

\section{Description of the structures}

The description and rationalization of (I) and (II) are based on the crystal structures obtained from the single-crystal diffraction data [from Dale \& Elsegood, 2003, for (II)], whereas discussion of structure (III) is based on that from the powder data.

\subsection{Molecular conformations}

3.1.1. Triethyl-1,3,5-triazine-2,4,6-tricarboxylate (I). The molecular conformation of (I) is planar (all non-H atoms lie on a mirror plane), and the molecule retains threefold molecular symmetry (Fig. 5). Displacement ellipsoids for all the non- $\mathrm{H}$ atoms are elongated in the direction perpendicular to the plane of the molecule, the largest elongation being that for $\mathrm{O} 2$. This clearly indicates the presence of disorder in the structure, with the short bond distances [C4-C5 = 1.423 (5) and $\mathrm{C} 2-\mathrm{O} 2=1.173(5) \AA$ ] also consistent with a disordered model. The planar symmetrical conformation in (I) is similar to that in both the tris(dimethylamino) derivative (Bullen et $a l ., 1972)$ and the $\gamma$-polymorph of the trimethoxy derivative (Fridman et al., 2004), the latter of which also displays elongation of the ellipsoids perpendicular to the molecular plane.

The conformation of the ethyl carboxylate side chains in (I) is also comparable to that seen in (II) [see Table 3 and Dale \& Elsegood (2003)] and in tris(2-hydroxyethyl)-1,3,5-benzene- 
tricarboxylate (Azumaya et al., 2004) in which there are only slight deviations from planarity with the aromatic ring.

3.1.2. Tris-2-hydroxyethyl isocyanurate (III). The molecular dimensions of (III) are similar to those obtained from powder refinements of analogous compounds, with intramolecular bond lengths and angles showing no unusual features (Fig. 6). The conformation of (III) is similar to that of the majority of other isocyanurate structures, with two of the hydroxyethyl groups oriented on one side of the heterocyclic ring, whilst the

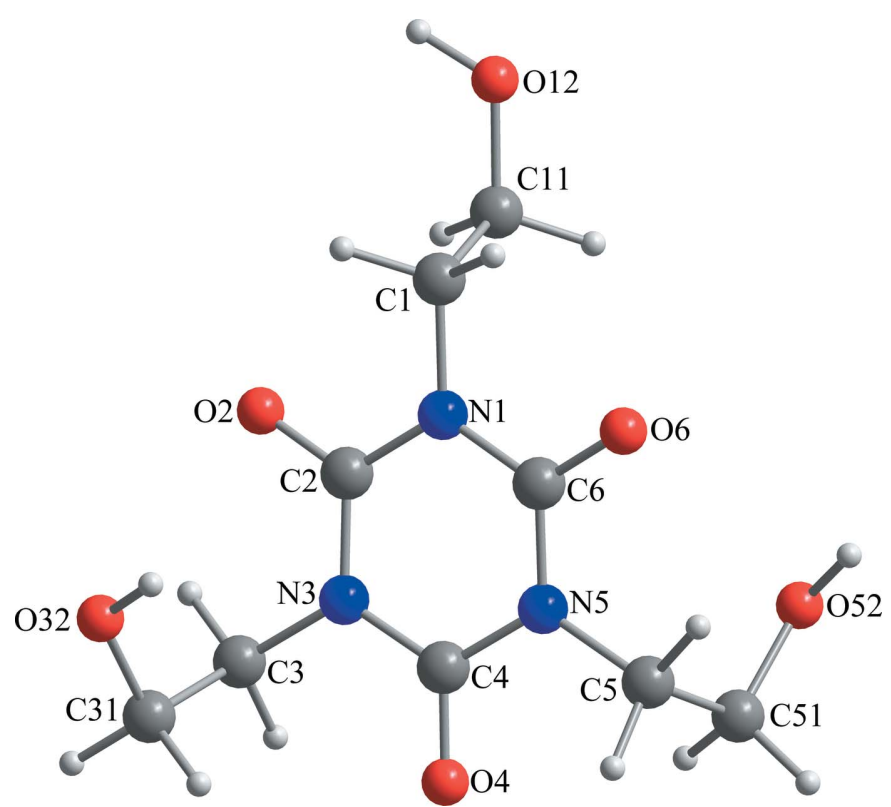

Figure 6

The refined molecular structure of (III), showing the conformation and atom-labelling scheme.

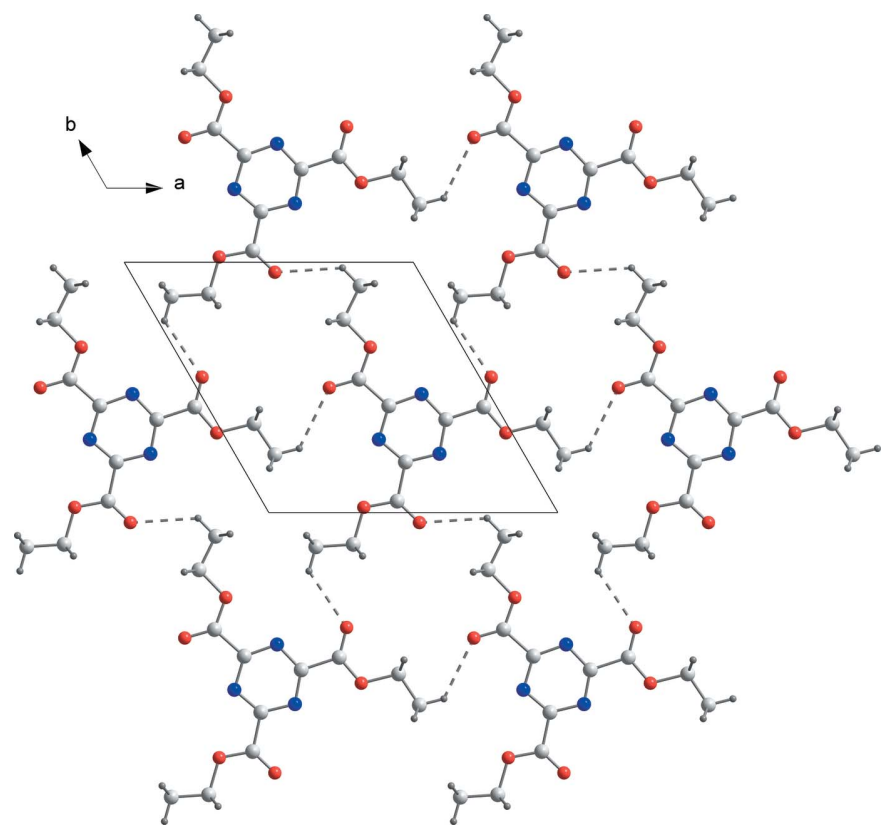

Figure 7

Part of the crystal structure of (I), showing a hydrogen-bonded sheet in the (001) plane. Hydrogen bonds are shown as dashed lines. third points in the other direction. Despite pointing in opposite directions with respect to the ring, two of these hydroxyethyl side chains have a similar conformation, whereas the third group, which is involved in more intermolecular hydrogen bonding than the other two (\$3.2.2), displays a conformation that is approximately perpendicular to the plane of the ring (Table 3 ).

\subsection{Supramolecular aggregation}

3.2.1. Triethyl-1,3,5-triazine-2,4,6-tricarboxylate (I). The supramolecular structure of (I) can be rationalized in terms of a single soft $\mathrm{C}-\mathrm{H} \cdots \mathrm{O}=\mathrm{C}$ hydrogen bond. Atom $\mathrm{C} 5$ at $(x, y$, $z$ ) acts as a donor via $\mathrm{H} 5 A$ to atom $\mathrm{O} 2$ at $(2-y, x-y, z)$, while atom $\mathrm{O} 2$ at $(x, y, z)$ acts as an acceptor of $\mathrm{H} 5 A$ at $(2-x$ $+y, 2-x, z)$. Propagation of this hydrogen bond with the threefold symmetry of the molecule $[(1-y, x-y, z)$ and $(1-x+y, 1-x, z)]$ means that each molecule is surrounded by six others. This results in the formation of a hydrogenbonded sheet parallel to (001), containing alternating $R_{3}^{3}(18)$ and $R_{3}^{3}(30)$ rings in a checkerboard pattern (Fig. 7). These

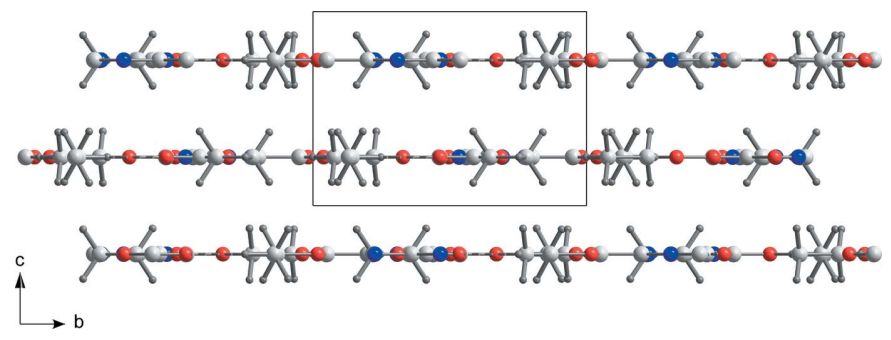

Figure 8

A view of (I) showing the stacking of layers in the [001] direction.

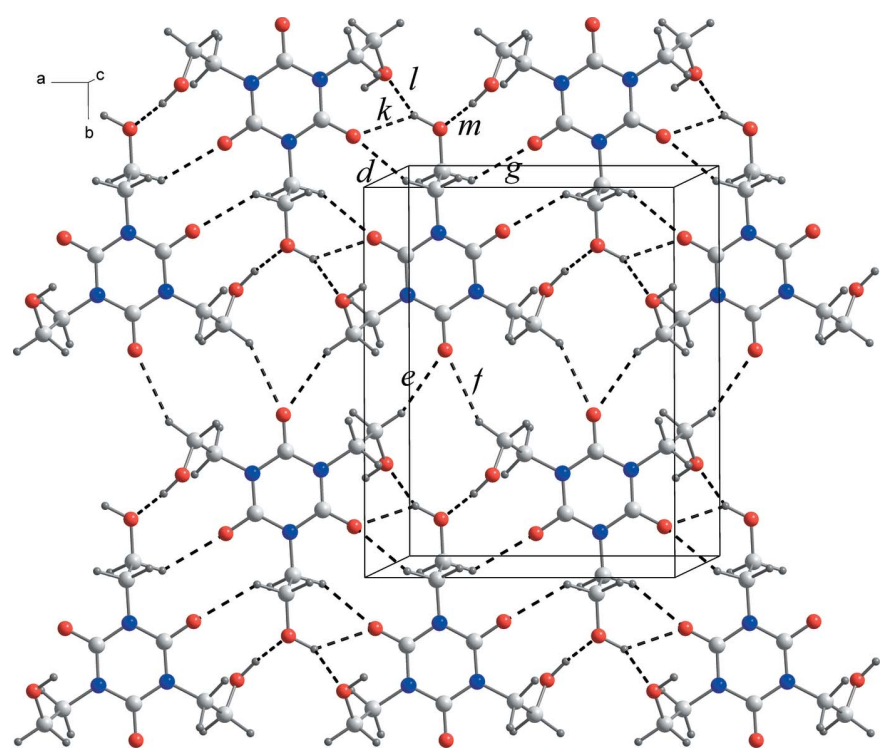

Figure 9

Part of the crystal structure of (III), showing a hydrogen-bonded layer in the (001) plane. Hydrogen bonds are shown as dashed lines, with the soft $\mathrm{C}-\mathrm{H} \cdots \mathrm{O}$ bonds indicated as $d, e, f$ and $g$, and the hard $\mathrm{O}-\mathrm{H} \cdots \mathrm{O}$ bonds as $k, l$ and $m$. 
Table 4

Intermolecular hydrogen-bond parameters $\left(\AA,^{\circ}\right)$ for (I)-(III).

\begin{tabular}{|c|c|c|c|c|c|}
\hline$D-\mathrm{H} \cdots A \dagger$ & $\mathrm{H} \cdots A$ & $D \cdots A$ & $D-\mathrm{H} \cdots A$ & $\begin{array}{l}\text { Motif } \\
\text { (basic) }\end{array}$ & $\begin{array}{l}\text { Motif } \\
\text { (higher) }\end{array}$ \\
\hline $\begin{array}{l}\text { Compound (I) } \\
\mathrm{C} 5-\mathrm{H} 5 A \cdots \mathrm{O} 2^{\mathrm{i}}\end{array}$ & $2.580(2)$ & $3.286(4)$ & $130.6(3)$ & $\begin{array}{l}R_{3}^{3}(18) \\
R_{3}^{3}(30)\end{array}$ & \\
\hline 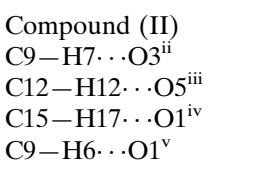 & $\begin{array}{l}2.811(2) \\
2.718(2) \\
2.831(2) \\
2.701(2)\end{array}$ & $\begin{array}{l}3.500(3) \\
3.431(5) \\
3.524(4) \\
3.664(4)\end{array}$ & $\begin{array}{l}128.1(2) \\
130.0(2) \\
128.4(2) \\
167.5(2)\end{array}$ & $\begin{array}{l}C(10) \\
C(10) \\
C(10) \\
C(6)\end{array}$ & $\begin{array}{l}R_{3}^{3}(18) \\
R_{3}^{3}(30)\end{array}$ \\
\hline 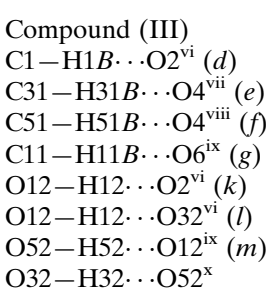 & $\begin{array}{l}2.498(6) \\
2.618(5) \\
2.670(4) \\
2.477(6) \\
2.285(5) \\
2.242(2) \\
1.756(3) \\
1.881(2)\end{array}$ & $\begin{array}{l}3.334(6) \\
3.659(5) \\
3.698(4) \\
3.310(5) \\
3.019(5) \\
2.981(3) \\
2.703(3) \\
2.802(3)\end{array}$ & $\begin{array}{l}132.4(2) \\
159.0(1) \\
151.6(1) \\
131.9(2) \\
131.5(2) \\
131.9(8) \\
165.5(1) \\
158.2(1)\end{array}$ & $\begin{array}{l}R_{2}^{2}(10) \\
R_{2}^{2}(12) \\
R_{2}^{2}(12) \\
R_{2}^{2}(12) \\
R_{2}^{2}(14) \\
R_{2}^{2}(20) \\
R_{2}^{2}(20) \\
C(10)\end{array}$ & \\
\hline
\end{tabular}

Symmetry codes: (i) $2-y, x-y, z$; (ii) $1+x, y, z$; (iii) $x, 1+y, z$; (iv) $-1+x,-1+y, z$; (v) $x-y,-x+y, \frac{1}{6}+z$; (vi) $2-x,-y,-z$; (vii) $2-x, 1-y,-z$; (viii) $1-x, 1-y,-z$; (ix) $1-x,-y,-z$; (x) $\frac{1}{2}+x, \frac{1}{2}-y, \frac{1}{2}+z$. $\dagger$ Lower-case italic letters in parentheses indicate hydrogen bonds denoted in Fig. 9.

sheets stack along the $c$ axis, at an interlayer distance of 3.382 (1) $\AA$ (Fig. 8). These layers are staggered by $x=-\frac{1}{3}, y=\frac{1}{3}$ such that intermolecular $R_{3}^{3}(18)$ rings lie directly above and below the triazine rings in alternate sheets.

Although triethyl-1,3,5-benzenetricarboxylate (II) in the structure of the molecule itself does not display any molecular or crystallographic symmetry, the crystal packing of (II) is similar to that of (I). The crystal structure of (II) is also controlled by $\mathrm{C}-\mathrm{H} \cdots \mathrm{O}$ interactions, and forms a hydrogenbonded sheet parallel to (001) in which each molecule is surrounded by six others, generating alternating $R_{3}^{3}(18)$ and $R_{3}^{3}(30)$ rings in a checkerboard pattern. There is an additional $\mathrm{C}-\mathrm{H}$. . O hydrogen- bond linking molecules from adjacent planes into helices around the $6_{1}$ axis in the [001] direction (Table 4).

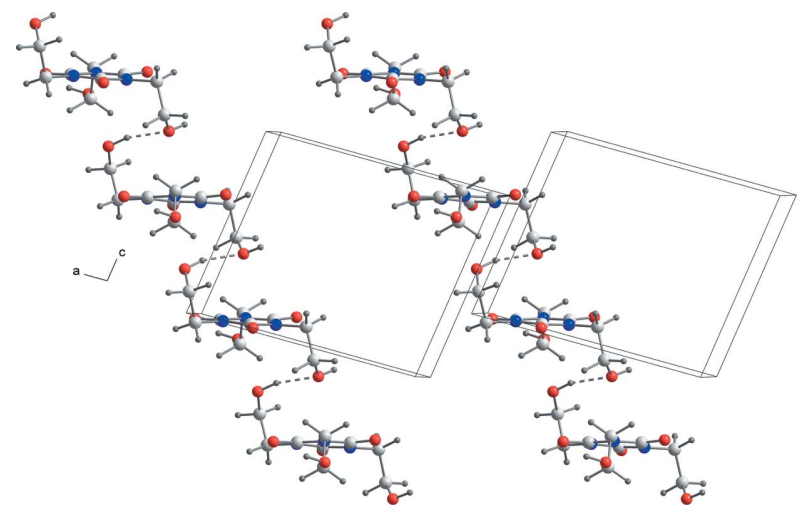

Figure 10

Stereoview of part of the crystal structure of (III), showing the $C(10)$ spiral chain parallel to [101]. Hydrogen bonds are shown as dashed lines.
3.2.2. Tris-2-hydroxyethyl isocyanurate (III). In (III) the supramolecular structure is determined by eight hydrogen bonds: four soft $\mathrm{C}-\mathrm{H} \cdots \mathrm{O}=\mathrm{C}$ hydrogen bonds and four hard hydrogen bonds, three of $\mathrm{O}-\mathrm{H} \cdots \mathrm{O}$ (hydroxyl) type and one $\mathrm{O}-\mathrm{H} \cdots \mathrm{O}=\mathrm{C}$ type (Table 4 ), such that all strong hydrogenbond donors and acceptors are utilized in the intermolecular network.

All but one of these hydrogen bonds are involved in the formation of a hydrogen-bonded sheet lying in the (001) plane. Each molecule is connected to four other molecules within the sheet via $\mathrm{C}-\mathrm{H} \cdots \mathrm{O}$ hydrogen bonds (denoted $d, e$, $f$ and $g$ ) with the interactions to two of these molecules reinforced by $\mathrm{O}-\mathrm{H} \cdots \mathrm{O}$ hydrogen bonds (denoted $k, l$ and $m$ ) (Fig. 9). The hydroxyl $\mathrm{O} 12$ at $(x, y, z)$ acts as a double hydrogen-bond donor, via $\mathrm{H} 12$, to carbonyl atom $\mathrm{O} 2$ (bond $k$ ) and hydroxyl $\mathrm{O} 32$ (bond $l)$ at $(2-x,-y,-z)$, while also acting as an acceptor from atom O52 via H52 (bond $m$ ) at (1$x,-y,-z)$. These interactions result in the formation of a ribbon running in the [100] direction, reinforced by atoms C1 and $\mathrm{C} 11$ in the molecule at $(x, y, z)$ acting as hydrogen-bond donors to carbonyl atoms $\mathrm{O} 2$ (bond $d$ ) at $(2-x,-y,-z$ ) [forming an $R_{2}^{2}(10)$ ring] and O6 (bond $g$ ) at $(1-x,-y,-z$ ) [forming an $R_{2}^{2}(12)$ ring]. The ribbons are then linked together into a hydrogen-bonded sheet through two $R_{2}^{2}(12)$ rings formed by atoms C31 and C51 at $(x, y, z)$ acting as hydrogenbond donors to $\mathrm{O} 4$ (bonds $e$ and $f$ ) in molecules $(2-x, 1-y$, $-z)$ and $(1-x,-y,-z)$, respectively (Fig. 9). These sheets are held together by a fourth strong hydrogen bond in which O32 at $(x, y, z)$ acts as a donor via $\mathrm{H} 32$ to $\mathrm{O} 52$ at $\left(\frac{1}{2}+x, \frac{1}{2}-y, \frac{1}{2}+z\right)$. This hydrogen bond produces a $C(10)$ chain motif running parallel to the [101] direction generated by the $n$-glide (Fig. 10).

Although the layer structure of (III) is distinct from the supramolecular packing seen in the majority of other symmetrically tri-substituted isocyanurate materials, the structure within the layers is almost identical to that in the crystal structure of the tris(2-cyanoethyl) derivative (Thallapally \& Desiraju, 2000).

\section{Concluding comments}

In this paper we have described the crystal structure determination of three tri-substituted molecular materials from conventional X-ray powder diffraction data. The difficulties encountered in the structure determination of two of these materials, (I) and (II), are not related to the traditional assessment of complexity based on the number of degrees of freedom for efficient direct-space optimization, but highlight more fundamental considerations for direct-space structure solution from powder diffraction, such as preferred orientation or deficiencies in the structural model. It is most likely that it is a combination of these factors that has prevented us from obtaining a good Rietveld profile fit for (I). In the case of (II) our study of the effect of side-chain conformation on the $R$ factor illustrates the dramatic effect that the consideration (or omission) of a preferred orientation correction can have on a fitness search surface and the resulting direct-space structure 
solution calculation. A successful optimization technique will only locate the global minimum of the surface that it explores, so it is the responsibility of the crystallographer to ensure that a suitable search surface is defined. In this case the use of soft geometrical restraints in refinement provided the side chains with the flexibility needed to adapt to the new $R$ factor surface defined by introduction of a preferred orientation correction during Rietveld refinement, thus preventing constraint of the molecule in a 'false' refinement minimum and potential consideration of a crystal structure with the incorrect molecular conformation.

The crystal structures resulting from this work display contrasting behaviour with respect to the retention of threefold molecular symmetry in crystal packing. Although the retention of this molecular symmetry is common in phenoxybased triazine derivatives (Thalladi et al., 1998, 1999), few other tri-substituted triazines display this behaviour. There are however, a small number of triazines, e.g. the triethynyl derivative (Ohkita et al., 2002) and the $\alpha$-form of the trimethoxy derivative (Fridman et al., 2004), that display the distinctive hexagonal-type layer packing seen in (I) without requiring the retention of threefold molecular symmetry in the crystal packing. In all these cases this 'local' acentric structural feature is not extended into the bulk, as the stacking of layers results in the overall structure being centrosymmetric. However, it is the similarity between the layer structure of the triazine (I) and its benzene analogue (II) that is distinct from previous comparisons of other systems. We believe that this is a consequence of the presence of sufficient hydrogen-bond donors and acceptors in the ethyl carboxylate side chains, and the resulting exclusion of heterocyclic $\mathrm{N}$ and aromatic $\mathrm{CH}$ atoms from the hydrogen-bond network within the layers of (I) and (II), respectively. Despite this, both systems maintain the characteristic trigonal non-centrosymmetric network with alternating 'unlike' substituents on neighbouring molecules pointing directly at each other. The lack of symmetry in the molecule of (II) allows the formation of an additional weak hydrogen-bonded helical motif between the layers and extension of non-centrosymmetry into the bulk structure. It is interesting to note that in all three comparative studies, the triazine-based materials display higher molecular symmetry in their crystal structure than the benzene-based equivalents. However, the possibility of polymorphism in these materials, and the differences observed in molecular symmetry between polymorphic forms of triazines (Fridman et al., 2004) and isocyanurates (Mariyatra et al., 2004) with $C_{3}$ molecular symmetry, makes the controlled design of materials through the transfer of such molecular symmetry to crystal symmetry a continuing challenge.

MT is grateful to the Royal Society for the award of a University Research Fellowship, and SYC thanks the University of Birmingham for financial support. CCS thanks the University of Birmingham and GlaxoSmithKline (UK) for studentship support.

\section{References}

Andreev, Y. G., Lightfoot, P. \& Bruce, P. G. (1996). Chem. Commun. pp. 2169-2170.

Azumaya, I., Uchida, D., Kato, T., Yokoyama, A., Tanatani, A., Takayanagi, H. \& Yokozawa, T. (2004). Angew. Chem. Int. Ed. 43, 1360-1363.

Brandenburg, K. (2005). DIAMOND. Version 3.1. Crystal Impact GbR, Bonn, Germany.

Bruker (2001). SAINT-Plus. Bruker AXS Inc., Madison, Wisconsin, USA.

Bullen, G. J., Corney, D. J. \& Stephens, F. S. (1972). J. Chem. Soc. Perkin 2, pp. 642-646.

Burnett, M. N. \& Johnson, C. K. (1996). ORTEPIII. Report ORNL6895. Oak Ridge National Laboratory, Tennessee, USA.

Cheung, E. Y., McCabe, E. E., Harris, K. D. M., Johnston, R. L., Tedesco, E., Raja, K. M. P. \& Balaram, P. (2002). Angew. Chem. Int. Ed. 41, 494-496.

Cordes, A. W., Haddon, R. C., Hicks, R. G., Kennepohl, D. K., Oakley, R. T., Schneemeyer, L. F. \& Waszczak, J. V. (1993). Inorg. Chem. 32, 1554-1558.

Dale, S. H. \& Elsegood, M. R. J. (2003). Acta Cryst. E59, o836-o837.

David, W. I. F., Shankland, K., McCusker, L. B. \& Baerlocher, Ch. (2002). Editors. Structure Determination from Powder Diffraction Data. Oxford University Press.

David, W. I. F., Shankland, K. \& Shankland, N. (1998). Chem. Commun. pp. 931-932.

Favre-Nicolin, V. \& Cerny, R. (2002). J. Appl. Cryst. 35, 734-743.

Fridman, N., Kapon, M., Sheynin, Y. \& Kaftory, M. (2004). Acta Cryst. B60, 97-102.

Harris, K. D. M., Tremayne, M. \& Kariuki, B. M. (2001). Angew. Chem. Int. Ed. 40, 1626-1651.

Harris, K. D. M., Tremayne, M., Lightfoot, P. \& Bruce, P. G. (1994). J. Am. Chem. Soc. 116, 3543-3547.

Johnston, J. C., David, W. I. F., Markvardsen, A. J. \& Shankland, K. (2002). Acta Cryst. A58, 441-447.

Kariuki, B. M., Serrano-Gonzalez, H., Johnston, R. L. \& Harris, K. D. M. (1997). Chem. Phys. Lett. 280, 189-195.

Larson, A. C. \& Von Dreele, R. B. (1987). GSAS. Generalized Structure Analysis System. Report No. LAUR-86-748. Los Alamos National Laboratory, New Mexico, USA.

Mariyatra, M. B., Panchanatheswaran, K., Low, J. N. \& Glidewell, C. (2004). Acta Cryst. C60, o682-o685.

Newsam, J. M., Deem, M. W. \& Freeman, C. M. (1992). Accuracy in Powder Diffraction II. NIST Special Publication No. 846, pp. 80-91. Gaithersburg, MA: NIST.

Ohkita, M., Kawano, M., Suzuki, T. \& Tsuji, T. (2002). Chem. Commun. pp. 3054-3055.

Pagola, S., Stephens, P. W., Bohle, D. S., Kosar, A. D. \& Madsen, S. K. (2000). Nature (London), 404, 307-310.

Panunto, T. W., Urbanczyk-Lipowska, Z., Johnson, R. \& Etter, M. (1987). J. Am. Chem. Soc. 109, 7786-7797.

Price, K. V. (1999). New Ideas in Optimization, edited by D. Corne, M. Dorigo \& F. Glover, pp. 77-158. London: McGraw-Hill.

Seaton, C. C. \& Tremayne, M. (2002a). Chem. Commun. pp. 880-881.

Seaton, C. C. \& Tremayne, M. (2002b). POSSUM. School of Chemistry, University of Birmingham, UK.

Sheldrick G. M. (1997). SHELXL97. University of Gottingen, Germany.

Shirley, R. A. (2000). CRYSFIRE. University of Surrey, UK.

Thalladi, V. R., Boese, R., Brasselet, S., Ledoux, I., Zyss, J., Jetti, R. K. R. \& Desiraju, G. R. (1999). Chem. Commun. pp. 1639-1640.

Thalladi, V. R., Brasselet, S., Blaser, D., Boese, R., Zyss, J., Nangia, A. \& Desiraju, G. R. (1997). Chem. Commun. pp. 1841-1842.

Thalladi, V. R., Brasselet, S., Weiss, H.-C., Blaser, D., Katz, A. M., Carrell, H. L., Boese, R., Zyss, J., Nangia, A. \& Desiraju, G. R. (1998). J. Am. Chem. Soc. 120, 2563-2577.

Thallapally, P. K. \& Desiraju, G. R. (2000). Acta Cryst. C56, 572-573. 


\section{research papers}

Tremayne, M. (2004). Philos. Trans. R. Soc. London Ser. A, 362, 2691-2707.

Tremayne, M., Seaton, C. C. \& Glidewell, C. (2002a). Am. Trans. 37, 35-50.
Tremayne, M., Seaton, C. C. \& Glidewell, C. (2002b). Acta Cryst. B58, 823-834.

Weiss, H.-C., Blaser, D., Boese, R., Doughan, B. M. \& Haley, M. M. (1997). Chem. Commun. pp. 1703-1704. 